\title{
Carotenogénesis de cinco cepas del alga Dunaliella sp. (Chlorophyceae) aisladas de lagunas hipersalinas de Venezuela
}

\author{
Miguel Guevara ${ }^{1 *}$, César Lodeiros ${ }^{1}$, Olga Gómez ${ }^{2}$, Nathalie Lemus ${ }^{1}$, Paulino Núñez ${ }^{1}$, Lolymar \\ Romero $^{1}$, Aléikar Vásquez ${ }^{1} \&$ Néstor Rosales ${ }^{3}$ \\ 1 Laboratorio de Acuicultura, extensión Plancton, Dpto. Biología Pesquera, Instituto Oceanográfico de Venezuela, \\ Universidad de Oriente, Cumaná 6101, Venezuela. \\ 2 Instituto de Investigaciones Científicas, Universidad de Oriente, Nueva Esparta, Venezuela. \\ 3 Departamento de Biología, Facultad Experimental de Ciencias, La Universidad del Zulia, Maracaibo, Venezuela. \\ *Autor de Correspondencia: Telf. 0293-4302118; miguevara2003@yahoo.com
}

\section{Received 08-IV-2005. Corrected 15-VI-2005. Accepted 06-VII-2005.}

\begin{abstract}
Carotenogenesis of five strains of the algae Dunaliella sp. (Chlorophyceae) isolated from Venezuelan hypersaline lagoons. We evaluated discontinuous cultures (Algal medium at $0.5 \mathrm{mM}$ of $\mathrm{NaNO}_{3}$, and $27 \% \mathrm{NaCl}$ ) of five strains of Dunaliella sp. isolated from Venezuelan hypersaline lagoons (Araya, Coche, Peonía, Cumaraguas, and Boca Chica) and one strain from a reference collection (Dunaliella salina, LB1644). Cultures were maintained to $25 \pm 1{ }^{\circ} \mathrm{C}$, with constant aeration, photoperiod 12:12, and two light intensities (195 and 390 $\mu \mathrm{E} . \mathrm{m}^{-2} \cdot \mathrm{s}^{-1}$ ) during 30 days. Cell count was recorded on a daily basis using a Neubaüer camera. Totals of chlorophyll $a$ and carotenoids were measured at the end of the experiment. The largest cellular densities were measured during the smallest light intensities. The strain with the largest cellular density was isolated from Boca Chica $\left(8 \times 10^{6}\right.$ and $2.5 \times 10^{6}$ cel.ml ${ }^{-1}$ a 390 and $195 \mu \mathrm{E} \cdot \mathrm{m}^{-2} \cdot \mathrm{s}^{-1}$, respectively). The increment of light intensity produced a significant reduction of growth rates in all strains. Totals of carotenoids by volume were as large as $390 \mu \mathrm{E} \cdot \mathrm{m}^{-2} \cdot \mathrm{s}^{-1}$. Strains LB1644, from Coche and Araya were those that produced the largest amount of carotenoids $(38.4 ; 32.8$ and 21.0 $\mu \mathrm{g} . \mathrm{ml}^{-1}$, respectively). Differences total carotenoids by cell between treatments were significant. The largest concentration was $390 \mu \mathrm{E} \cdot \mathrm{m}^{-2} \cdot \mathrm{s}^{-1}$. The strains LB1644 and Coche produced the highest values of carotenes (137.14 and 106.06 pg.cel ${ }^{-1}$, respectively). Differences in the relation carotenoid:chlorophyll $a$ between the strains at various light intensities was significant. Strains LB1644 presented the largest value of the relation carotenoids:chlorophyll $a(20: 1)$ at $195 \mu \mathrm{E} \cdot \mathrm{m}^{-2} \cdot \mathrm{s}^{-1}$. No significant differences were detected in the strain Coche (15:1). All the other strains showed relations lower than one. Our results suggest that the strains of Coche and Araya show potential to be used in the biotechnology of carotenoids production. Rev. Biol. Trop. 53(3-4): 331-337. Epub 2005 Oct 3.
\end{abstract}

Key words: Dunaliella, pigments, carotenoids, Venezuela.

Dunaliella salina es un alga clorofita predominante en ambientes hipersalinos, caracterizada por acumular grandes cantidades de carotenoides, llegando hasta $14 \%$ de su masa seca; lo que la convierte en una fuente potencial de $\beta$-caroteno natural de importancia para el uso en las industrias alimenticias y farmacéuticas, así como una herramienta útil para estudios bioquímicos relacionados con biosíntesis de pigmentos y mecanismos de regulación ante ambientes extremos (Borowitzka y Borowitzka 1988, Cifuentes et al. 1992, Gómez et al. 1992, Masuda et al. 2002).
Diversos estudios han demostrado que la producción de carotenoides en $D$. salina puede optimizarse mediante variaciones de las condiciones de cultivo, las cuales incluyen aumento de la salinidad, intensidad luminosa, temperatura (Ben-Amotz 1987, Gómez et al. 2003) y/o limitación de nutrientes (Borowitzka y Borowitzka 1988, Borowitzka et al. 1990, Mendoza et al. 1999).

En el trópico americano y específicamente en Venezuela los estudios con microalgas marinas se han dirigido, principalmente, hacia la producción de alimento para los primeros 
estadios larvarios de organismos acuáticos, siendo escasas las investigaciones relacionadas con la evaluación de cepas de $D$. salina potencialmente carotenogénicas, entre las que destaca la de Marín et al. (1998), quienes evaluaron una cepa de Dunaliella procedente de las salinas de Araya, estado Sucre, Venezuela, cultivada a bajas intensidades luminosas y diferentes salinidades y concentraciones de nitrato. En la presente investigación, como una forma de propiciar el cultivo de cepas autóctonas de Dunaliella productoras de carotenos, se evaluó la carotenogénesis y el crecimiento de cinco cepas de este género, provenientes de lagunas hipersalinas de las costas venezolanas, cultivadas a dos intensidades luminosas y elevada salinidad.

\section{MATERIALES Y MÉTODOS}

Organismos y condiciones de cultivo: Se utilizaron cinco cepas de Dunaliella sp., pertenecientes al cepario del Laboratorio de Acuicultura, extensión Plancton, del Instituto Oceanográfico de Venezuela, aisladas de salinas de las costas venezolanas (salinas de Araya, $10^{\circ} 37^{\prime} \mathrm{N}$; $64^{\circ} 7^{\prime} \mathrm{W}$, estado Sucre; salinas de Coche, $10^{\circ} 47^{\prime} \mathrm{N}$; 63⒌' $\mathrm{W}$, y laguna de

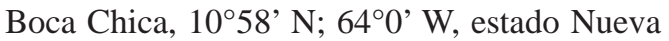
Esparta; salinas de las Peonías, $10^{\circ} 43^{\prime} \mathrm{N}$; 71³5' $\mathrm{E}$, estado Zulia y salinas de las Cumaraguas, $12^{\circ} 06^{\prime} \mathrm{N}$; 6954' W, estado Falcón) y una cepa de colección, Dunaliella salina LB1644, utilizada como referencia.

Las microalgas fueron cultivadas en agua de mar con una elevada salinidad $(27 \% \mathrm{NaCl}$ w. $\mathrm{v}^{-1}$ ) filtrada (filtros $\mathrm{GF} / \mathrm{F}$ ), esterilizada en autoclave $\left(120^{\circ} \mathrm{C} / 15 \mathrm{~min} / 15 \mathrm{psi}\right)$ y enriquecida con medio Algal (Fábregas et al. 1986) con una concentración de $0.5 \mathrm{mM}$ de nitrato.

Los cultivos (2 1, por triplicado) con aireación constante a $200 \mathrm{ml} \cdot \mathrm{min}^{-1}$, se iniciaron con $8 \times 10^{3}$ cel. $\mathrm{ml}^{-1}$ y se sometieron a dos intensidades luminosas (195 y $390 \mu \mathrm{E} . \mathrm{m}^{-2} . \mathrm{s}^{-1}$ ) durante 30 días, con un fotoperiodo 12:12 y una temperatura de $25 \pm 1^{\circ} \mathrm{C}$. Antes de iniciar los ensayos, y como mecanismo para aislar sólo Dunaliella salina, las cepas se cultivaron en un medio deficiente de fosfatos, Provasoli, durante 15 días (McLachlan 1973) y luego se aclimataron durante 30 días a las mismas condiciones ambientales mencionadas anteriormente.

Crecimiento celular y análisis de pigmentos: La densidad celular (cel.ml-1) de los cultivos se determinó diariamente mediante conteos de alícuotas en una cámara de Neubaüer. Las tasas crecimiento se calcularon en la fase de crecimiento exponencial mediante la relación establecida en Madigan et al. (1999).

Las muestras para los análisis de pigmentos ( $5 \mathrm{ml}$, por triplicado) se colectaron al final del ensayo y se centrifugaron a $4000 \mathrm{rpm}$ durante 15 min. La clorofila $a$ y carotenoides totales se extrajeron a partir de las microalgas sedimentadas, utilizando una mezcla acetona:agua $90 \%$. La determinación se realizó por espectrofotometría (Wegmann y Metzner 1971).

Análisis estadísticos: Los datos se analizaron a través de un análisis de varianza factorial, considerando como factores las cepas y las intensidades luminosas. Cuando se determinaron diferencias significativas entre los tratamientos, se aplicó la prueba Scheffé $(\alpha=0.05)$ de comparaciones múltiples entre medias (Sokalf y Rolf 1995).

\section{RESULTADOS}

Crecimiento: La Fig. 1 muestra el crecimiento de las seis cepas de Dunaliella sp. Las mayores densidades celulares en todas las cepas ocurrieron a la menor intensidad luminosa ensayada. La cepa que alcanzó las mayor densidad celular fue la de Boca Chica ( $8 \pm$ 0.403 × $10^{6}$ y $2.5 \pm 0.202 \times 10^{6}$ cel. $\mathrm{ml}^{-1}$ a 195 y $390 \mu \mathrm{E} \cdot \mathrm{m}^{-2} \cdot \mathrm{s}^{-1}$, respectivamente). En general, la tendencia de crecimiento en las curvas fue de incremento acelerado durante los cinco primeros días, indicando la fase logarítmica, para luego alcanzar y mantenerse más o menos a una tasa constante, excepto el último día cuando se evidencia el inicio de la fase estacionaria. Esta tendencia es más marcada en los organismos 

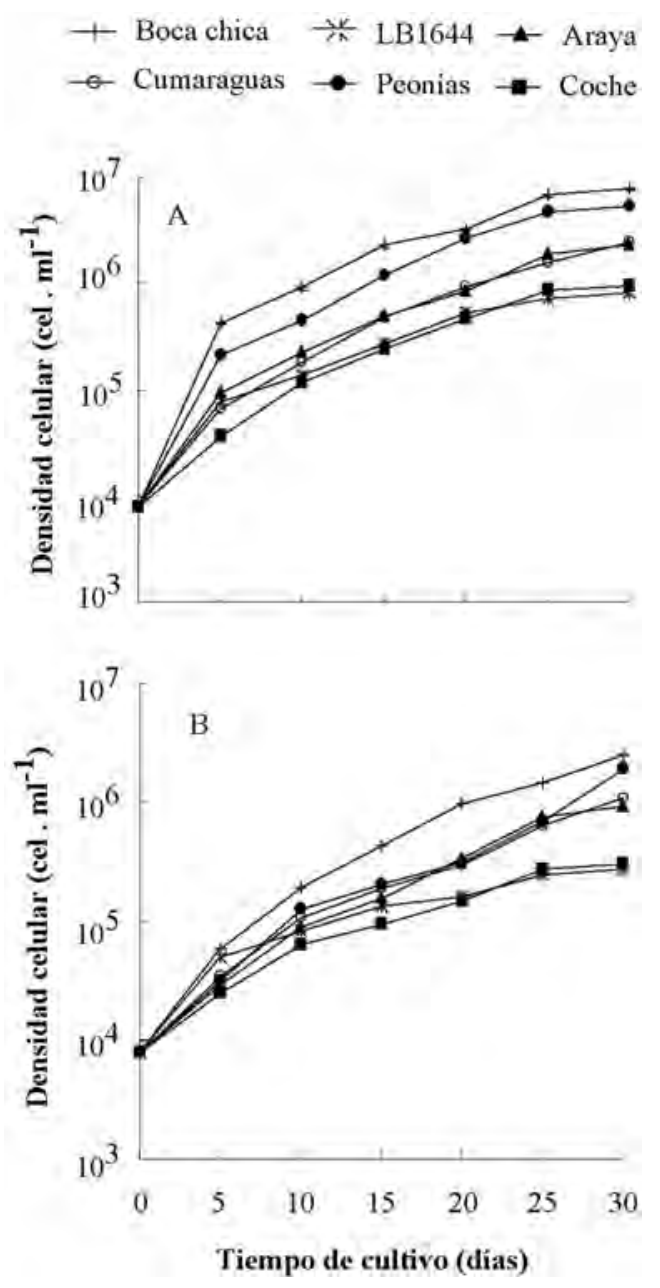

Fig. 1. Crecimiento de seis cepas de Dunaliella sp. cultivadas a $27 \% \mathrm{NaCl}(\mathrm{w} / \mathrm{v})$, durante 30 días a 195 (A) y 390 (B) $\mu \mathrm{E} / \mathrm{m}^{2} / \mathrm{s}$.

Fig. 1. Growth of six strains of Dunaliella sp. cultivated to $27 \% \mathrm{NaCl}(\mathrm{w} / \mathrm{v})$, during 30 days at $195(\mathrm{~A})$ and $390(\mathrm{~B})$ $\mu \mathrm{E} / \mathrm{m}^{2} / \mathrm{s}$.

cultivados a la menor intensidad de luz. El incremento de la intensidad luminosa en los cultivos produjo una disminución significativa de las tasas de crecimiento, particularmente con las cepas de Coche y la cepa de referencia (Fig. 2). Entre las cepas se determinaron diferencias significativas $(\mathrm{p}<0.001)$, donde: Araya $=$ Cumaragua $=$ Peonía $>$ Coche $=$ Boca Chica > LB644.

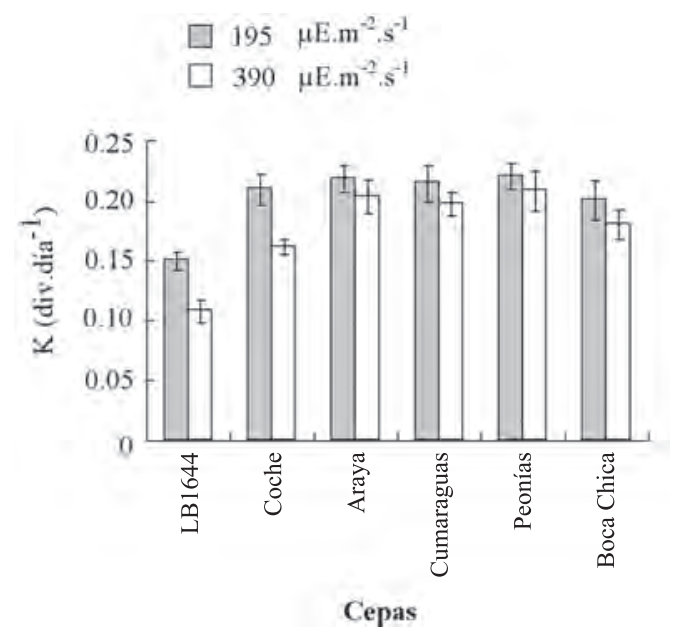

Fig. 2. Tasas de crecimiento instantáneo ( \pm desviación estándar), en fase exponencial, de seis cepas de Dunaliella sp. cultivadas a $27 \% \mathrm{NaCl}(\mathrm{w} / \mathrm{v})$, durante 30 días a 195 y $390 \mu \mathrm{E} / \mathrm{m}^{2} / \mathrm{s}$.

Fig. 2. Rates of instantaneous growth $( \pm$ standard deviation), in exponential phase, of six strains of Dunaliella sp. cultivated to $27 \% \mathrm{NaCl}(\mathrm{w} / \mathrm{v})$, during 30 days at 195 y $390 \mu \mathrm{E} / \mathrm{m}^{2} / \mathrm{s}$.

Clorofila $a$ : El contenido de clorofila a por volumen $\left(\mu \mathrm{g} \cdot \mathrm{ml}^{-1}\right)$ mostró diferencias significativas $(\mathrm{p}<0.001)$ en las seis cepas y en las dos intensidades luminosas ensayadas (Fig. 3A). Las cepas LB1644 y Coche mostraron concentraciones más altas de este pigmento a $390 \mu \mathrm{E} \cdot \mathrm{m}^{-2} \cdot \mathrm{s}^{-1}$, mientras que en las cepas Araya, Cumaragua, Peonía y Boca Chica se obtuvieron a $195 \mu \mathrm{E} \cdot \mathrm{m}^{-2} \cdot \mathrm{s}^{-1}$.

El contenido de clorofila $a$ por célula (pg.cel ${ }^{-1}$ ) presentó diferencias significativas $(\mathrm{p}<0.001)$ entre las intensidades luminosas y entre las cepas. A $390 \mu \mathrm{E} \cdot \mathrm{m}^{-2} \cdot \mathrm{s}^{-1}$ se produjo la mayor concentración de este pigmento (Fig. 3B). Entre las cepas, LB1644 y Coche produjeron más del doble de este pigmento, particularmente a la mayor intensidad de luz.

Carotenoides totales: Los análisis de varianza mostraron diferencias significativas $(p<0.001)$ entre los tratamientos en el contenido de carotenoides totales por volumen $\left(\mu \mathrm{g} \cdot \mathrm{ml}^{-1}\right)$. La mayor concentración de este pigmento, en 

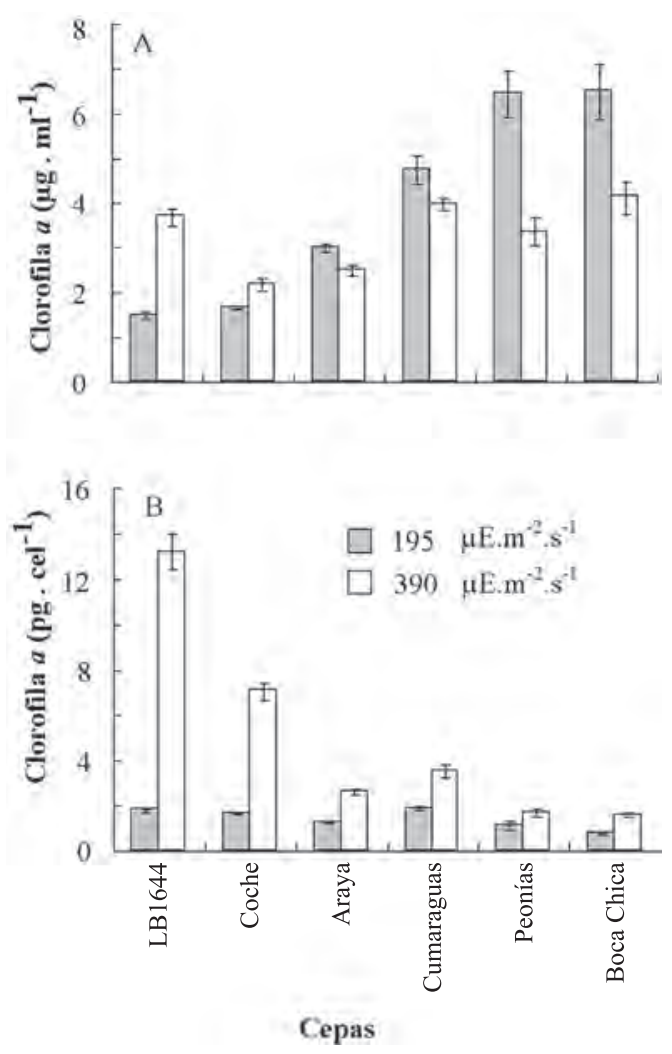

Fig. 3. Contenido promedio ( \pm desviación estándar) de clorofila $a$ en $\mathrm{g} / \mathrm{ml}$ (A) y pg/cel (B) de seis cepas de Dunaliella sp. cultivadas a $27 \% \mathrm{NaCl}(\mathrm{w} / \mathrm{v})$, durante 30 días a 195 y $390 \mu \mathrm{E} / \mathrm{m}^{2} / \mathrm{s}$.

Fig. 3.Mean content ( \pm standard deviation) of chlorophyll $a$ in $\mu \mathrm{g} / \mathrm{ml}$ (A) and pg/cel (B) of six strains of Dunaliella sp. cultivated to $27 \% \mathrm{NaCl}(\mathrm{w} / \mathrm{v})$, during 30 days at 195 and $390 \mu \mathrm{E} / \mathrm{m}^{2} / \mathrm{s}$.

todas las cepas, se obtuvo a $390 \mu \mathrm{E} \cdot \mathrm{m}^{-2} \cdot \mathrm{s}^{-1}$, siendo las cepas LB1644, Coche y Araya las que produjeron mayor cantidad, con concentraciones de $38.40 \pm 1.920 ; 32.88 \pm 1.970$ y $21.00 \pm$ $0.840 \mu \mathrm{g} \cdot \mathrm{ml}^{-1}$, respectivamente (Fig. 4A).

Para el caso del contenido de carotenoides totales por célula (pg.cel $\left.{ }^{-1}\right)$, éstos presentaron diferencias significativas por efecto de la intensidad luminosa (Fig. 4B), observándose que la mayor concentración se registró a 390 $\mu \mathrm{E} . \mathrm{m}^{-2} \cdot \mathrm{s}^{-1}$; siendo las cepas LB1644 y la de Coche las que produjeron los valores más altos de carotenos $(137.14 \pm 6.850$ y $106.06 \pm$
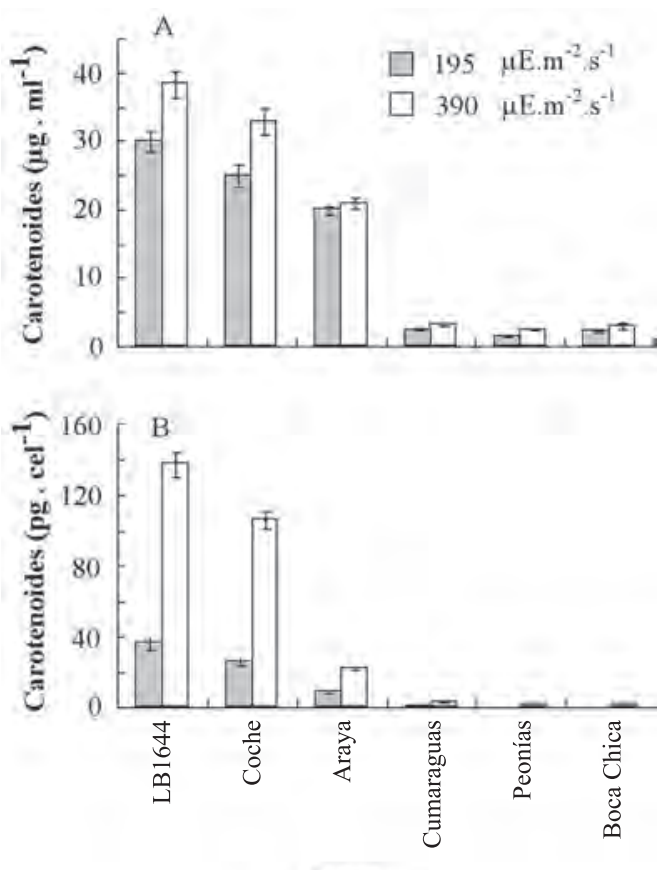

Cepas

Fig. 4. Contenido promedio ( \pm desviación estándar $)$ de carotenoides totales en $\mathrm{g} / \mathrm{ml}$ (A) y $\mathrm{pg} /$ cel (B) de seis cepas de Dunaliella sp. cultivadas a $27 \% \mathrm{NaCl}(\mathrm{w} / \mathrm{v})$, durante 30 días a 195 y $390 \mu \mathrm{E} / \mathrm{m}^{2} / \mathrm{s}$.

Fig. 4. Mean content ( \pm standard deviation) of total carotenoids in $\mu \mathrm{g} / \mathrm{ml}$ (A) and $\mathrm{pg} / \mathrm{cel}$ (B) of six strains of Dunaliella sp. cultivated to $27 \% \mathrm{NaCl}(\mathrm{w} / \mathrm{v})$, during 30 days at 195 and $390 \mu \mathrm{E} / \mathrm{m}^{2} / \mathrm{s}$.

5.300 pg.cel ${ }^{-1}$, respectivamente), duplicando los obtenidos por las restantes cepas.

La relación carotenoides totales:clorofila $a$ mostró diferencias significativas entre las cepas y las intensidades luminosas ensayadas. La cepa LB1644 presentó la mayor relación (20.0 \pm 1.200 ) a $195 \mu \mathrm{E} \cdot \mathrm{m}^{-2} \cdot \mathrm{s}^{-1}$, mientras que en la cepa de Coche no se evidenciaron diferencias significativas entre las dos intensidades, obteniéndose una relación de $15.15 \pm 1.060$. La cepa de Araya alcanzó la mayor relación (8.40 $\pm 0.756)$ a $390 \mu \mathrm{E} \cdot \mathrm{m}^{-2} \cdot \mathrm{s}^{-1}$. El resto de las cepas mostraron relaciones inferiores a uno (Fig. 5). 


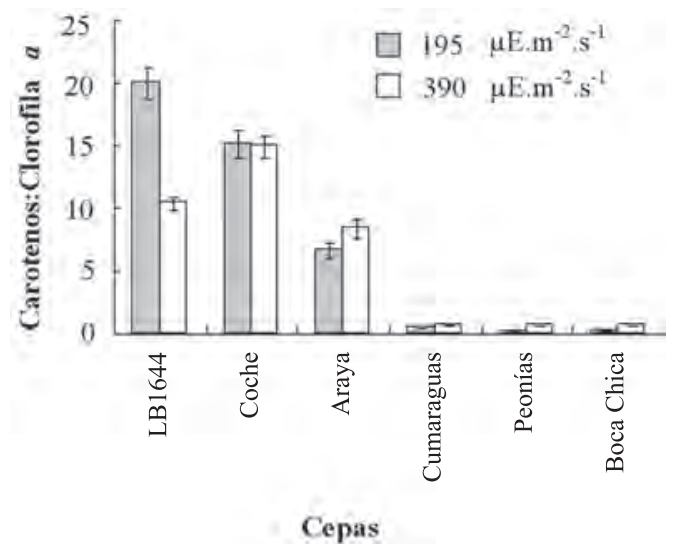

Fig. 5. Relación carotenoides: clorofila a ( \pm desviación estándar) de seis cepas de Dunaliella sp. cultivadas a $27 \%$ $\mathrm{NaCl}(w / v)$, durante 30 días a 195 y $390 \mu \mathrm{E} / \mathrm{m}^{2} / \mathrm{s}$.

Fig. 5. Total carotenoides to chlorophyll $a$ ratio ( \pm standard deviation) of six strains of Dunaliella sp. cultivated to $27 \%$ $\mathrm{NaCl}(\mathrm{w} / \mathrm{v})$, during 30 days at 195 and $390 \mu \mathrm{E} / \mathrm{m}^{2} / \mathrm{s}$.

\section{DISCUSIÓN}

En esta investigación se evidenció el efecto que tienen las condiciones de cultivo estresantes (altas salinidades e intensidades luminosas y deficiencia de nitrógeno) sobre el crecimiento y la carotenogénesis de las cepas estudiadas, lo cual ha sido observado en otras cepas de Dunaliella (Ben-Amotz y Avron 1983, Borowitzka y Borowitzka 1988). Estos factores indujeron la carotenogénesis en las cepas LB1644, Coche y Araya. El resto de las cepas (Cumaragua, Peonías y Boca Chica) mostraron una disminuida capacidad carotenogénica.

La capacidad de producción de carotenoides de las cepas de Coche y Araya probablemente fue generada evolutivamente por las condiciones ambientales estresantes que presentan las salinas de donde fueron aisladas, las cuales poseen temperaturas extremas $\left(27-37^{\circ} \mathrm{C}\right)$, altas intensidades luminosas y salinidades entre $30-35 \%$ de $\mathrm{NaCl}$, lo que favorece la proliferación de un reducido número de especies de microorganismos; entre éstos Dunaliella salina, que pigmenta las aguas con un tono rojizo intenso. En este sentido, Cifuentes et al. (1992) informaron que las condiciones ambientales de un hábitat determinado pueden, en parte, ser las responsables del desarrollo de la carotenogénesis en una cepa particular de D. salina. Esta hipótesis se encuentra soportada con nuestros resultados, ya que las cepas Araya y Coche fueron obtenidas en muestras de agua con concentraciones salinas superiores a $30 \%$ de $\mathrm{NaCl}$ y las restantes cepas a concentraciones inferiores a $20 \%$. Una hipótesis alternativa es que las cepas Araya y Coche son tipo Dunaliella salina y las restantes tipo Dunaliella viridis; la evidencia de un menor tamaño de estas últimas sugiere que las cepas son especies diferentes. Por su parte, la cepa de referencia (LB1644) aportó los mejores resultados de carotenoides en esta investigación, ratificando así su validada productividad de carotenoides.

Las altas concentraciones de carotenoides totales por volumen y por célula producidas por las cepas de Coche $\left(38.88 \pm 1.97 \mu \mathrm{g} \cdot \mathrm{ml}^{-1}\right.$ ó $106.06 \pm 5.300$ pg.cel $\left.{ }^{-1}\right)$ y Araya $(21.00 \pm$ $0.84 \mu \mathrm{g} \cdot \mathrm{ml}^{-1}$ ó $22.10 \pm 0.88$ pg.cel ${ }^{-1}$ ), así como la relación carotenoides:clorofila $a$, sugieren el potencial carotenogénico que presentan estas cepas. Los valores obtenidos por la cepa Coche son superiores a los citados por Cifuentes et al. (1992), 8.1-42.4 pg.cel ${ }^{-1}$ para cepas provenientes de Chile (Antofagasta y Atacama); Marín et al. (1998), 0.8-1.6 pg.cel ${ }^{-1}$ en cepas de las salinas de Araya, Venezuela; Gómez et al. (2003), 47.7 y 26.9 pg.cel ${ }^{-1}$ para cepas aisladas de Chile (Atacama) y Egipto (Bardawil Lake), respectivamente, pero menores que los informados por Cifuentes et al. (1996), 124 pg.cel ${ }^{-1}$ para otra cepa aislada del salar de Atacama (Chile). A pesar de que se establecen comparaciones entre el contenido de carotenoides de diferentes cepas de $D$. salina, resulta difícil analizarlas en todos sus aspectos, debido a que las respuestas en el crecimiento de esta microalga, como lo informa Ben-Amotz (1980), están influenciadas por una serie de factores que interactúan entre sí.

El aumento de la concentración de carotenoides totales en la mayor intensidad luminosa ensayada, puede deberse a mecanismos de fotoprotección de la célula, como lo sugieren 
Ben-Amotz y Avron (1983). Estos pigmentos pueden alcanzar mayores concentraciones si se limita la disponibilidad de nitrógeno en el medio de cultivo, lo cual pudo haber ocurrido en esta investigación, debido a que se utilizó una baja concentración de nitratos $(0.5 \mathrm{mM})$. En este sentido, Loeblich (1982); Ben-Amotz (1987) y Marín et al. (1998) indicaron que el aumento de carotenoides y el cambio en la tonalidad de los cultivos se hace evidente en células estresadas por deficiencia de nutrientes.

En relación a los resultados obtenidos, las cepas Araya y, particularmente, la de Coche pueden ser consideradas como microorganismos potenciales para su uso en la biotecnología en la producción de carotenoides. Estudios analíticos de pigmentos, haciendo énfasis en el tipo de carotenoides, son sugeridos para determinar dicha potencialidad para ser usadas como productoras de carotenoides en actividades de acuicultura. Con respecto a las cepas Cumaraguas, Peonías y Boca Chica, se hace necesario un estudio más exhaustivo para corroborar su taxonomía ya que, a pesar de haber crecido previamente en el medio Provasoli (McLachlan 1973) a altas intensidades luminosas y salinidades, que permite obtener cultivos unialgales de Dunaliella salina, muestran bajas concentraciones celulares de carotenos.

\section{AGRADECIMIENTOS}

Este estudio fue financiado por el FONACIT (S1 2000001599) y por el Consejo de Investigación de la Universidad de Oriente (CI-5-1802-1154/03).

\section{RESUMEN}

Se realizaron cultivos discontinuos (medio Algal con $0.5 \mathrm{mM}$ de $\mathrm{NaNO}_{3}$ y $27 \%$ de $\mathrm{NaCl}$ ) de cinco cepas de Dunaliella sp., aisladas de diferentes lagunas hipersalinas de Venezuela (Araya, Coche, Peonía, Cumaraguas y Boca Chica) y una cepa de referencia (Dunaliella salina LB1644). Los bioensayos se mantuvieron a $25 \pm 1{ }^{\circ} \mathrm{C}$ con aireación constante, fotoperiodo 12:12 y dos intensidades luminosas (195 y $390 \mu \mathrm{E} \cdot \mathrm{m}^{-2} \cdot \mathrm{s}^{-1}$ ) durante 30 días. El crecimiento celular se determinó diariamente mediante conteo celular en cámara de Neubaüer. La clorofila $a$ y los carotenoides totales se analizaron al final del ensayo. Las mayores densidades celulares correspondieron a los ensayos de menor intensidad luminosa. La cepa que alcanzó la mayor densidad celular fue la aislada de Boca Chica $(8$ x $10^{6}$ y $2.5 \times 10^{6}$ cel. $^{-1} l^{-1}$ a 195 y $390 \mu \mathrm{E} . \mathrm{m}^{-2} \cdot \mathrm{s}^{-1}$, respectivamente). El incremento de la intensidad luminosa en los cultivos produjo una disminución significativa de las tasas de crecimiento en todas las cepas. Los carotenoides totales por volumen fueron mayores a $390 \mu \mathrm{E} \cdot \mathrm{m}^{-2} . \mathrm{s}^{-1}$; siendo las cepas de referencia LB1644, Coche y Araya las que produjeron mayor cantidad $\left(38.4 ; 32.8\right.$ y $21.0 \mu \mathrm{g} . \mathrm{ml}^{-1}$, respectivamente). El contenido de carotenoides totales por célula en los dos tratamientos fue significativamente diferente, obteniéndose la mayor concentración a $390 \mu \mathrm{E} \cdot \mathrm{m}^{-2} \cdot \mathrm{s}^{-1}$. Las cepas LB1644 y Coche fueron las que produjeron los valores más altos de carotenos (137.14 y 106.06 pg.cel $^{-1}$, respectivamente). La cepa LB1644 presentó la mayor relación carotenoides totales:clorofila $a(20: 1)$ a $195 \mu \mathrm{E} \cdot \mathrm{m}^{-2} \cdot \mathrm{s}^{-1}$, mientras que en la cepa Coche no se evidenciaron diferencias significativas entre las dos intensidades (15:1). El resto de las cepas mostraron relaciones inferiores a uno. Nuestros resultados sugieren que las cepas Coche y Araya pueden ser potencialmente utilizadas en la biotecnología de producción de carotenoides.

Palabras clave: Dunaliella, pigmentos, carotenoides, Venezuela.

\section{REFERENCIAS}

Ben-Amotz, A. 1980. Glycerol production in the alga Dunaliella, p. 191-208. In A. San Pietro (ed.). Biochemical and photosynthetic aspects of energy production. Academic, Nueva York, Nueva York.

Ben-Amotz, A. 1987. Effect of irradiance and nutrient defienciency on the chemical composition of Dunaliella bardawil Ben-Amotz and Avron (Volvocales, Chlorophyta). J. Plant Physiol. 131: 479-487.

Ben-Amotz, A. \& M. Avron.1983. On the factors which determine massive $\beta$-carotene accumulation in the halotolerant alga Dunaliella bardawil. Plant Physiol. 72: 593-597.

Borowitzka, M. \& L. Borowitzka. 1988. Dunaliella, p. 27-58. In M. Borowitzka \& L. Borowitzka (eds.). Microalgal Biotechnology. Cambridge University, Cambridge, Inglaterra.

Borowitzka, M., L. Borowitzka \& D. Kessly. 1990. Effects of salinity increase on carotenoid accumulation in the green alga Dunaliella salina. J. Appl. Phycol. 2: 111-119. 
Cifuentes, A., M. González, M. Conejeros, V. Dellarossa \& O. Parra. 1992. Growth and carotenogenesis in eight strains of Dunaliella salina Teodoresco from Chile. J. Appl. Phycol. 4: 111-118.

Cifuentes, A., M. González, O. Parra \& M. Zúñiga. 1996. Cultivo de cepas de Dunaliella salina (Teodoresco 1905) en diferentes medios bajo condiciones de laboratorio. Rev. Chil. Hist. Nat. 69: 105-112.

Fábregas, J., C. Herrero, B. Cabezas, R. Liaño \& J. Abalde. 1986. Response of the marine microalga Dunaliella tertiolecta to nutrient concentration and salinity variations in batch cultures. J. Plant Physiol. 125: 475-484.

Gómez, J., Z. Ramazanov, A. Fontes \& G. García. 1992. Photosynthetic characteristics of Dunaliella salina (Chlorophyceae, Dunaliellales ) in relation to $\beta$-carotene content. J. Appl. Phycol. 4: 11-15.

Gómez, P., A. Barriga, A. Cifuentes \& M. González. 2003. Effect of salinity on the quantity and quality of carotenoids accumulated by Dunaliella salina (strain CONC-007) and Dunaliella bardawil (strain ATCC 30861). Biol. Res. 36: 185-192.

Loeblich, L.1982. Photosynthesis and pigments influenced by light intensity and salinity in the halophile Dunaliella salina (Chlorophyta). J. Mar. Biol. Ass. UK. 62: 493-508.
Madigan, M., J. Martinko \& J. Parker. 1999. Brock Biología de los microorganismos.. Prentice Hall Iberia, Madrid, España.1064 p.

Marín, N., F. Morales, C. Lodeiros \& E. Tamigneaux. 1998. Effect of nitrate concentration on growth and pigments synthesis of Dunaliella salina cultivated under low illumination and preadapted to different salinities. J. Appl. Phycol. 10: 405-411.

Masuda, T., J. Polle \& A. Mellis. 2002. Biosynthesis and distribution of chlorophyll among the photosystems during recovery of the green alga Dunaliella salina from irradiance stress. Plant Physiol. 128: 603-614.

Mc Lachlan, J. 1973. Growth media-marine, p. 25-51. In J. Stein (ed.). Handbook of phycological methods. Culture methods and growth measurements. Cambridge University, Cambridge, Inglaterra.

Mendoza, H., A. Martel, M. Jiménez \& M. García. 1999. Oleic acid is the main fatty acid related with carotenogenesis in Dunaliella salina. J. Appl. Phycol. 11: 15-19.

Sokal, R. \& F. Rohlf. 1995. The principles and practice of statistics in biological research. Third edition. W. H. Freeman, Nueva York. 887 p.

Wegmann, K. \& H. Metzner. 1971. Synchronization of Dunaliella salina cultures. Arch. Mikrobiol. 78: 360-367. 
